\title{
Anisotropy and dynamics of photospheric velocity patterns: 2D power and coherence analyses
}

\author{
A. Nesis, R. Hammer, H. Schleicher, and M. Roth
}

Kiepenheuer-Institut für Sonnenphysik, Schöneckstr. 6, 79104 Freiburg, Germany

e-mail: nesis@kis.uni-freiburg.de

Received 5 July 2011 / Accepted 21 January 2012

\section{ABSTRACT}

\begin{abstract}
Context. The dynamical and topological properties of a fluid define its hydrodynamical state and energy transfer. By means of twodimensional (2D) spectroscopy and 2D power and coherence analyses we study these properties in the solar photosphere.

Aims. To obtain insight into the change of the velocity field with height in the solar photosphere we analyze 2D spectroscopic observations.

Methods. Maps of the vertical velocity at four different photospheric heights are studied by means of 2D power and coherence analyses, in order to characterize the dynamical and topological properties of the velocity field in the 2D wave number domain $\left(k_{x}, k_{y}\right)$. (i) The power analysis shows the power amplitude and its distribution over the $\left(k_{x}, k_{y}\right)$ domain for each velocity map and thus height level. We use the mean azimuthal presentation to provide a quick 1D overview. (ii) The cross-amplitude spectrum shows interrelationships between two velocity maps. We use the cross-amplitude spectrum to visualize and quantify changes of the velocity patterns with height in the photosphere. (iii) The square coherence is the normalized cross power spectrum; it represents the correlation in the $\left(k_{x}, k_{y}\right)$ domain. The degree of isotropy of this quantity signifies the existence of velocity patterns with different shapes. To facilitate the visualization of the $2 \mathrm{D}$ power and coherence maps we calculate their $1 \mathrm{D}$ mean azimuthal values.

Results. The 2D power and coherence analyses reveal that the velocity fields of the higher photospheric layers are different from the deeper granular layers. The loss of similarity is found to occur in the mid photosphere. The highest photospheric layers are characterized by (i) a diminution of the velocity power; (ii) a disappearance of the small velocity structures; and (iii) a tendency for larger upflow velocity structures to become asymmetric.
\end{abstract}

Key words. Sun: photosphere - Sun: granulation

\section{Introduction}

The photospheric velocity field consists of granular-like flows, wave motions, and intermittent velocity bursts. In order to improve our understanding of this complex dynamical system it is important to study the variation of granular velocity patterns with height.

Spectrograms of high spectral resolution involving absorption lines of various strengths provide a proper way to study the dynamical and spatial properties of the granular flow with height. Historically the work around this topic started with a paper by Canfield \& Mehltretter (1973) and continued in papers by Nesis (1985), Roudier \& Muller (1986), Nesis \& Mattig (1989), Komm et al. (1990, 1991), Roudier et al. (1991), Espagnet et al. (1993), and Hanslmeier et al. (2000). In contrast to this earlier work based on one-dimensional (1D) spectra, two-dimensional (2D) spectroscopy offered the opportunity to observe granular shapes and velocity patterns in the solar photosphere without the need for spatial scans.

Espagnet et al. (1995) investigated the vertical structure of the photosphere based on series of $2 \mathrm{D}$ multichannel subtractive double pass (MSDP) spectrograms. They found that most of the granular intensity structures disappear between 65 and $90 \mathrm{~km}$ above $\tau_{500}=1$. The associated granular velocity fluctuations, however, cross the entire thickness of the photosphere. They also showed that the weak pattern of intensity fluctuations present above $90 \mathrm{~km}$ is not connected to the granular pattern.

Making use of the Göttingen Fabry-Pérot interferometer (GFPI) at the Vacuum Tower Telescope (VTT) at the
Observatorio del Teide, Krieg et al. (1999) studied the disappearance of the granular intensity structures with height and found that "granular intensity fluctuations are a matter of the deep photosphere alone".

To investigate the dynamics of the solar photosphere down to small granular scales, Hirzberger et al. (2001) combined spectroscopy with image reconstruction techniques. They analyzed spectral scans of narrow-band images across the non-magnetic Fe I $557.6 \mathrm{~nm}$ line with the GFPI at the VTT. Concerning granular intensity and velocity fluctuations they found that for structures larger than one arcsec the intensity fluctuations vanish rapidly with photospheric height, whereas the flow velocities stay almost constant up to $250 \mathrm{~km}$. In another paper Hirzberger (2002) investigated statistically the intensity and velocity patterns at different photospheric levels. Applying new and innovative concepts from fractal geometry statistics to the analysis of granular patterns he found that the overall granular shapes are significantly elongated. Furthermore Hirzberger (2002) showed that the temporal coherence decreased according to a power-law with an exponent that is incompatible with the Kolmogorov energy spectrum of homogeneous and isotropic turbulence. He interpreted this as observational evidence against an overall turbulent character of granular motions.

Berrilli et al. (2002) studied particularly the stratification aspect of the velocity and intensity fluctuations of the granulation at the solar disk center. Analyzing time series of broad band and monochromatic images they investigated the damping of convective motions in stably stratified layers by means of larger granules passing through a large part of the photosphere. 
Their results suggest the occurrence of an intense braking within the first $120 \mathrm{~km}$ above $\tau_{500}=1$. The observations were obtained with the IPM observing mode of the THEMIS telescope (Observatorio del Teide, Tenerife), which consists of a Universal Birefringent Filter followed by a Fabry-Pérot interferometer. To study velocity and intensity patterns at different heights in the photosphere they used the absorption lines C I $538.02 \mathrm{~nm}$, $\mathrm{Fe}$ I $537.96 \mathrm{~nm}$ and Fe I $557.61 \mathrm{~nm}$.

Puschmann et al. (2005) analyzed a time series of high resolution slit spectrograms. They found a similar distribution of velocity and temperature structures with height and interpreted this as observational evidence for substantial overshoot into the photosphere.

All of the above mentioned publications manifest the division of the photosphere into two distinct layers with different physical properties: in the lower photosphere, the granular intensity patterns of convective origin lose their similarity gradually within the first $150 \mathrm{~km}$ above $\tau_{500}=1$. On the other hand, the upper photosphere seems to be governed by a flow with constant velocity amplitude and velocity patterns that begin to deviate from those of the lower region. This different behavior of intensity and velocity structures, although both are of convective origin, calls for a detailed determination of the velocity patterns at different heights in the photosphere.

In a recent paper, Rieutord et al. (2010) used Hinode data to discuss the properties of the photospheric dynamics from subto supergranular scales. They investigated horizontal flows by feature tracking and vertical velocities spectroscopically, at two height levels.

In the present paper we study topological and dynamical properties in the photosphere with particular emphasis on the height variation of the vertical velocity pattern. For this purpose we obtained a series of $2 \mathrm{D}$ spectrograms of high spectral resolution to produce maps of the radial velocity representing four different height layers. The data are processed by means of $2 \mathrm{D}$ power and coherence analyses.

2D power and coherence analyses are evidently the proper methods for investigating changes of the velocity patterns in the frequency domain. If the topology of a velocity map is the same for the $x$ - and $y$-axis, the 2D power function is mirror symmetric with respect to the diagonal. An asymmetry, on the other hand, would indicate that the signals in both directions are not the same. This is e.g. the case when the velocity field shows elongated patterns.

Power analysis allows us to study topological and dynamical properties of each of the four velocity maps individually. Although this gives us some information about the variation of the velocity field with height, it cannot reveal the full interrelationships between the fields represented by the various velocity maps. In order to describe not only the properties of the individual velocity maps but also the "cross links" that may exist between them, we introduce the cross covariance (or cross correlation functions) and cross spectra. The normalized cross spectrum is the square coherence. In the 2D case it can reveal photospheric plasma properties in the frequency domain like, for example, the isotropic behavior of its velocity field.

\section{Observation and method}

\subsection{Observation and data acquisition}

The investigation is based on a series of 2D spectroscopic observations of a magnetically insensitive iron line. The observational parameters are given in Table 1. For each scan, image distortions
Table 1. Observational parameters.

\begin{tabular}{ll}
\hline \hline Spectral line & Fe I 557.66 nm \\
Date of observations & 2007-June-30 \\
Telescope & VTT at Izaña, Tenerife \\
Start of first scan & UT 8:05 \\
End of last scan & UT 8:46 \\
Location at Sun & disk center \\
\hline Spectrograph: & high resolution mode \\
TESOS $(*)$ & $1.9 \mathrm{pm}$ \\
FPI bandwidth & $0.83 \mathrm{pm}$ \\
FPI scanning step width & 101 \\
number of steps per scan & $83 \mathrm{pm}$ \\
scan range & $50 \mathrm{~ms}$ \\
exposure time & $\approx 20 \mathrm{~s}$ \\
scan duration $(1$ profile $)$ & $25 \mathrm{~s}$ \\
scanning cadence & 100 \\
number of scans & \\
\hline
\end{tabular}

Notes. (*) Triple Etalon Solar Spectrometer, Tritschler et al. (2002).

Table 2. Formation heights for the bisector levels.

\begin{tabular}{lccc}
\hline \hline $\begin{array}{l}\text { Line } \\
\text { depression }\end{array}$ & $h_{\text {lower }}$ & $h_{\max }$ & $h_{\text {upper }}$ \\
\hline 0.2 & 10 & 75 & 150 \\
0.5 & 40 & 100 & 180 \\
0.75 & 70 & 140 & 350 \\
1.0 & 345 & 420 & 540 \\
\hline
\end{tabular}

Notes. The line depressions are relative to the central depression of the line (see also Fig. 1). $h_{\max }$ is the height in $\mathrm{km}$ where the contribution function of the line depression is at maximum, $h_{\text {lower }}$ and $h_{\text {upper }}$ are the lower and upper heights where the contribution function is at half maximum.

were determined from the white light images, which are obtained simultaneously with the images from the Fabry-Pérot interferometer (FPI) channel. A destretching algorithm was applied to remove distortions in the FPI images. From the 101 FPI images of each scan, line profiles were constructed for each image pixel, normalized to the continuum intensity.

Maps of the line-of-sight velocity were constructed by measuring the Doppler shift of the three bisector levels 0.2, 0.5, 0.75, and of the line center "1.0" (see Fig. 1). The formation heights of the three bisector levels and the line core are shown in Table 2. In the next step we removed the effect of the p-mode oscillations by applying a $(k / \omega)$ Fourier filter to the time series. In this way we obtained non-oscillatory velocity maps at the four heights in the photosphere (see Table 2). The size of the useful field of view (FOV) is $20 \times 20 \mathrm{Mm}$.

For this investigation, we selected the best four scans of the series to minimize the influence of seeing. They were obtained at $2.25,8.92,16.00$, and $30.00 \mathrm{~min}$, respectively, after the beginning of the observation. For these scans, the achieved spatial resolution is about $0.3 \operatorname{arcsec}(220 \mathrm{~km})$, covered by about 3.5 CCD camera pixels. We start by analyzing in detail the first of these scans, at $2.25 \mathrm{~min}$; thereafter we investigate the coherence between all four selected scans.

\subsection{Data processing}

In the following, the terms $u^{(0.2)}, u^{(0.5)}$, and $u^{(0.75)}$ denote the velocity maps for Doppler shift measurements at the bisector levels $0.2,0.5,0.75$, respectively. The term $u^{(1.0)}$ denotes the 


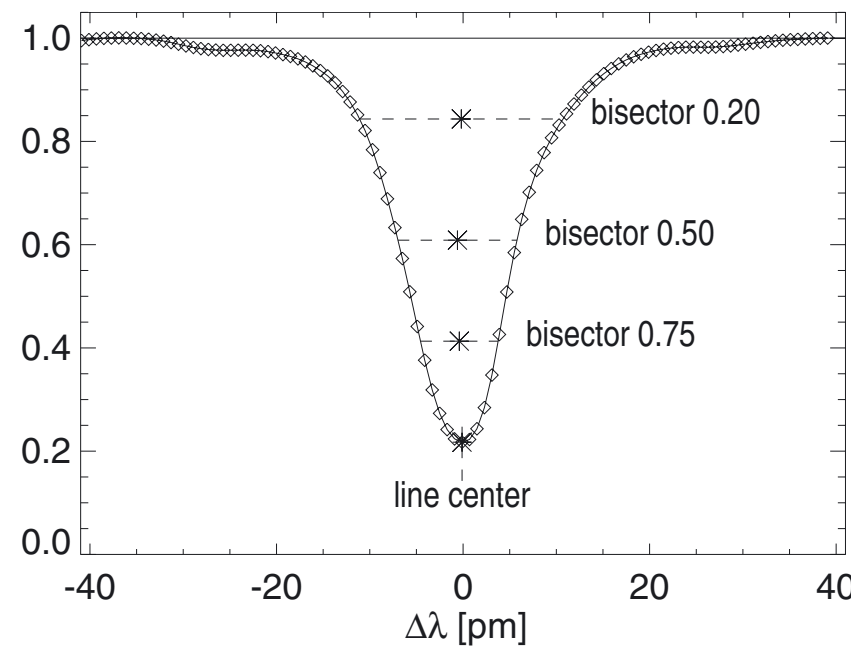

Fig. 1. Mean profile of the nonmagnetic line Fe I $557.6 \mathrm{~nm}$. The values associated with each bisector level are the line depression relative to the line center depression. The three selected bisector levels and the line center probe four different heights in the solar atmosphere.

velocity map for Doppler shift measurement at the line center. The four velocity maps represent the dynamical conditions of the photosphere at heights $h_{\max }$ of $75,100,140$, and $420 \mathrm{~km}$ above $\tau_{500}=1$. However, as is demonstrated in Table 2, the response functions are overlapping.

To gain insight into the photospheric dynamics and its topological aspects we subject the velocity maps to a 2D power, cross covariance, and coherence analysis. The 2D power gives us information about the dynamical and topological properties of each of the four levels in the photosphere. The 2D coherence analysis is based on the $2 \mathrm{D}$ cross covariance, which reflects a single input-single output relation: it relates the output, i.e. the maps $u^{(0.5)}, u^{(0.75)}, u^{(1.0)}$, to the input velocity map $u^{(0.2)}$. Thus, it describes the velocity relationship between the bisector levels 0.2 and $0.5,0.2$ and 0.75 , and 0.2 and 1.0 , respectively, in the frequency domain.

In order to provide an overview of the $2 \mathrm{D}$ power, cross power and coherence functions we remove the directional information in these functions by averaging over the azimuth around the origin of wave number space.

The fundamentals of $1 \mathrm{D}$ power and coherence analyses are treated in various textbooks, e.g. Stearns \& David (1993) and Priestley (1999). In Appendix A we briefly summarize the important equations and definitions extended to their $2 \mathrm{D}$ form.

\section{Results and discussion}

\subsection{White light images and spectroscopic velocity maps}

Figure 2 shows the continuum image together with the maps of radial velocities derived from the Doppler shifts at bisector levels 0.2 and 0.75 (relative line depressions, Fig. 1) for one of the 101 scans. The two maps $u^{(0.2)}, u^{(0.75)}$ represent the radial velocity field at about $75 \mathrm{~km}$ and $140 \mathrm{~km}$ above the continuum emitting layer. Upflows and downflows are color coded by blue and red, respectively. In the $u^{(0.2)}$ velocity map, probing deep layers in the photosphere, the green-blue patterns correspond to the bright parts of the granulation seen in the continuum image. This correspondence is weaker for the $u^{(0.75)}$ velocity map, probing higher layers, where only the strongest upflows appear as green-blue patches.
There is a strong similarity between the morphology of the $u^{(0.2)}$ velocity map and the white light image in Fig. 2 . This similarity begins to weaken at larger photospheric height as shown by comparing the $u^{(0.75)}$ velocity map with the white light image. The velocity map $u^{(0.75)}$ shows generally less velocity structuring; especially the small-scale blue-green and red structures in Fig. 2 are reduced in number. This means that small-scale velocity fluctuations do not reach the higher layers. These upper layers are dominated by plasma flows of small velocity amplitudes (yellow) and upflows with large amplitudes (blue-green) that are organized predominantly in large, sometimes elongated, velocity structures.

All these results confirm that the velocity patterns in the upper photospheric layers are organized in a different manner than those in the deep photospheric layers. The latter reflect the pattern organization of the upper boundary layers of the convective zone. In the following we quantify these observable phenomena by means of $2 \mathrm{D}$ velocity power spectra.

\section{2. $2 D$ spatial velocity power spectra}

In Fig. 3 the four panels and their insets show the results of the power analysis applied to the four velocity maps $u^{(0.2)}, u^{(0.5)}$, $u^{(0.75)}, u^{(1.0)}$. They demonstrate the variation of dynamical and topological features with height in the photosphere. Since we are interested only in relative changes, the power is given in arbitrary units in the current and the subsequent figures.

Let us first consider the mean azimuthal power function $P_{\mathrm{a}}$ shown in the insets of Fig. 3. We realize (i) the shrinking of the area under the functions $P_{\mathrm{a}}^{(0.75)}$ and $P_{\mathrm{a}}^{(1.0)}$, (ii) the shift of the azimuthal power function towards smaller spatial frequencies with increasing height, and (iii) the similarity between $P_{\mathrm{a}}^{(0.2)}$ and $P_{\mathrm{a}}^{(0.5)}$. The shrinking of the power area results from the reduction of the velocity amplitude and reflects thus a change of the photospheric dynamics with height. The shift of the wavenumber at which the power drops below a certain threshold reveals a change of the spatial scales in the velocity field and thus the change of its topological features with height. The similarity of the mean azimuthal power proves the extension of the granular behavior from the deepest layers considered (bisector level 0.2) to those corresponding to bisector level 0.5 . In the layers above that the power associated with small wavenumbers is reduced drastically.

In Fig. 3 the $2 \mathrm{D}$ power spectra, the $P^{(0.2)}, P^{(0.5)}, P^{(0.75)}$, and $P^{(1.0)}$ power maps, show that at a certain bisector level between 0.5 and 0.75 there is a change in dynamics and morphology, i.e. in the distribution of the spatial scales of the patterns of the velocity field. The morphological change is reflected by the behavior of the contour of constant power in the power maps: the $P^{(0.75)}$ and $P^{(1.0)}$ maps have a reflectional symmetry with respect to the diagonal through the origin in the $k_{x}-k_{y}$ plane, in contrast to the maps $P^{(0.2)}$ and $P^{(0.5)}$. The dynamical change, on the other hand, is reflected by the mean azimuthal power.

We emphasize that the concentration of the power at scales larger than $\approx 2.0 \mathrm{Mm}$ in the power maps $P^{(0.75)}$ and $P^{(1.0)}$ is due to the disappearance of the small scale velocity fluctuations in the higher photospheric layers, a fact that is also demonstrated by the shift of the azimuthal power function towards smaller spatial frequencies with increasing height. This result is important because it implies that the dynamics of the upper photosphere is dominated by velocity fluctuations organized at scales larger than $\approx 2.0 \mathrm{Mm}$. This is also indicated in Fig. 2 . 

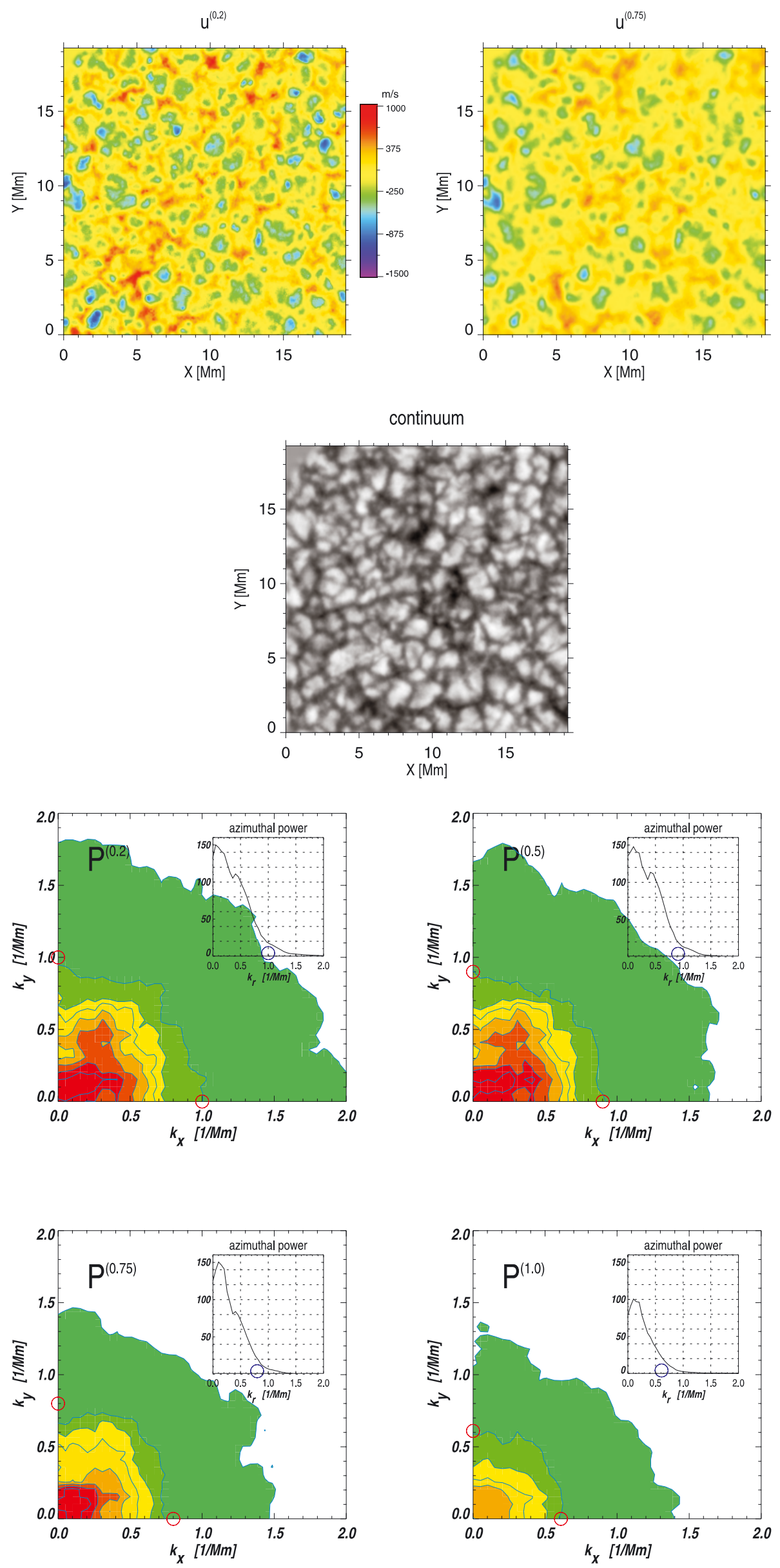

Fig. 2. Continuum image together with the maps of radial velocities derived from the Doppler shifts at bisector levels 0.2 and 0.75 (relative line depressions, see Fig. 1) for one of the selected scans (No. 5, taken 2.25 min after the start of the series). The color coding for the velocities is shown in the middle of the upper row.

Fig. 3. 2D spatial power spectra of the velocity fields measured at four bisector levels, for the same scan as shown in Fig. 2. The up per row shows two power maps corresponding to the velocity fields measured at the bisectors 0.2 (left) and 0.5 (right). The second row corresponds to the velocity fields measured at the bisectors 0.75 (left) and line core (right). The axes are the wave numbers $k_{x}$ and $k_{y}$. The power is color coded from red (maximum power) to green (minimum power). The insets show the corresponding mean azimuthal power $P_{\mathrm{a}}$. The small circles mark the wave number at which the signal disappears below a certain common threshold. 

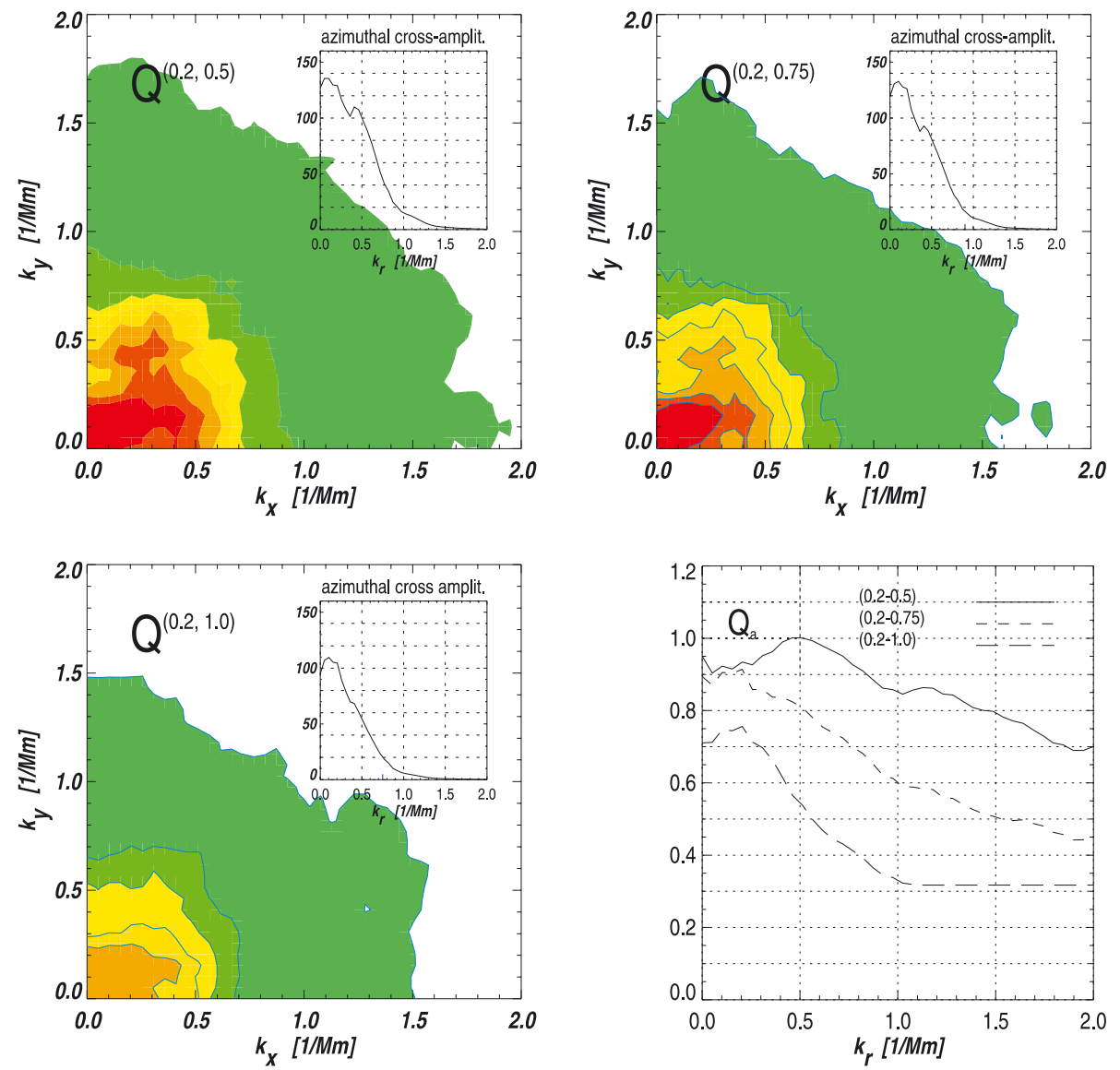

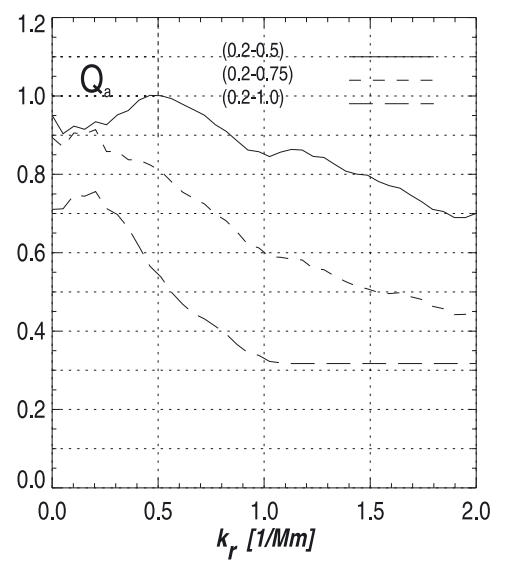

Fig. 4. 2D velocity cross-amplitude power $Q^{(0.2,0.5)}, Q^{(0.2,0.75)}$, and $Q^{(0.2,1.0)}$, derived from the corresponding velocity cross spectra, at the same time step as in Fig. 2. The cross spectra are obtained by correlating the velocity measurements at the line bisector level 0.2 and the velocity measurements at the bisector levels $0.5,0.75$, and line core, respectively. The axes of these first three panels represent the wave numbers $k_{x}$ and $k_{y}$. The cross amplitude power in these $2 \mathrm{D}$ spectra is indicated by different colors from red (maximum power) to green (minimum power). The insets show the corresponding mean azimuthal cross-amplitude power $Q_{a}\left(k_{r}\right)$. The lower right panel shows the three mean azimuthal cross amplitude spectra $Q_{a}^{(0.2,0.5)}$ (full line), $Q_{a}^{(0.2,0.75)}$ (dashed), and $Q_{a}^{(0.2,1.0)}$ (long dashed), respectively, normalized to the mean azimuthal velocity power at the line bisector $0.2, P_{a}^{(0.2)}$.
The part of the absorption line between bisector levels 0.5 and 0.75 (Fig. 1) originates largely in the first $120 \mathrm{~km}$ above $\tau_{500}=1$. So, our findings of a change in dynamics and in topology of the velocity field above these layers is in line with the disappearance of the granular intensity structures with height in the photosphere (Espagnet et al. 1995; Krieg et al. 1999; Berrilli et al. 2002; Hirzberger 2002).

While Espagnet et al. (1995) have already pointed out that velocity fluctuations associated with the granulation may cross the entire photosphere, our results show that this crossing is most efficient for the largest spatial scales, since with increasing height the velocity power is shifted towards smaller spatial frequencies in Fig. 3.

\section{3. $2 D$ velocity cross amplitude spectra}

The 2D cross amplitude spectra in Fig. 4 are obtained by correlating $u^{(0.2)}$ (granular layers) with $u^{(0.5)}, u^{(0.75)}$ and $u^{(1.0)}$ (higher layers), respectively. They show the power that the cross correlated velocity maps have in common at specific wavenumbers.

The cross amplitude power $Q^{(0.2,0.5)}$ is again not reflectionally symmetric with respect to the diagonal through the origin in the $k_{x}-k_{y}$ plane, especially in the $[0.2,0.5] \mathrm{Mm}^{-1}$ wavenumber interval. The mean azimuthal cross amplitude power function $Q_{\mathrm{a}}$ becomes small near a radial wavenumber of $1 \mathrm{Mm}^{-1}$, corresponding to a spatial scale of $\approx 1.3$ arcsec, indicating that structures with scales smaller than this value are no longer in common between the layers being compared. The $Q^{(0.2,0.75)}$ map is nearly identical to $Q^{(0.2,0.5)}$. The cross amplitude power $Q^{(0.2,1.0)}$ illustrates the relationship between the velocity fields at the deep and highest photospheric layers. At a first glance we notice a shift of the mean azimuthal power to smaller radial wavenumber $k_{r}$ (larger spatial scales), from $\approx 1.0 \mathrm{Mm}^{-1}$ to $\approx 0.75 \mathrm{Mm}^{-1}$. This corresponds to a shift by $0.5 \operatorname{arcsec}$ (from 1.3 arcsec to 1.8 arcsec).

The 2D cross amplitude power maps (Fig. 4) are useful to assess the transfer of a common power and the changing organization of the velocity patterns with increasing height in the photosphere. We first consider the mean azimuthal cross amplitude power functions shown in the insets. For the upper two panels these functions are very similar. This is a worthwhile finding since it indicates that the dynamics of the granular velocity fields and their topology remains the same up to layers corresponding to the 0.75 bisector level. The overlying layers, however, experience a clear change of the velocity field: the mean azimuthal cross amplitude power $Q_{\mathrm{a}}^{(0.2,1.0)}$ shows a strong diminution from 150 to 100 and a shift towards smaller wavenumbers (larger spatial scales). This implies that only $\approx 2 / 3$ of the power that the velocity maps $u^{(0.2)}, u^{(0.5)}$, and $u^{(0.75)}$ have in common reach the layers corresponding to the line core. The strong diminution of the power transfer from the 0.2 to the 1.0 bisector level is associated with the vanishing of the small scale velocity fluctuations in the layers corresponding to the line core (Figs. 2 and 3).

The results concerning the three mean azimuthal cross amplitude power functions and their variation with height are only partially in line with the results of earlier 2D-spectroscopic investigations. Most of the papers mentioned in the introduction conclude that the velocity fluctuations of the deep photospheric layers extend up to the higher photospheric layers. Only Berrilli et al. (2002) found that the granular velocity associated with large granules reaches only up to $\approx 120 \mathrm{~km}$ above $\tau_{500}=1$. This is similar to our results showing that the dynamics and topology of the velocity field change between the 0.75 and 1.0 bisector level. 

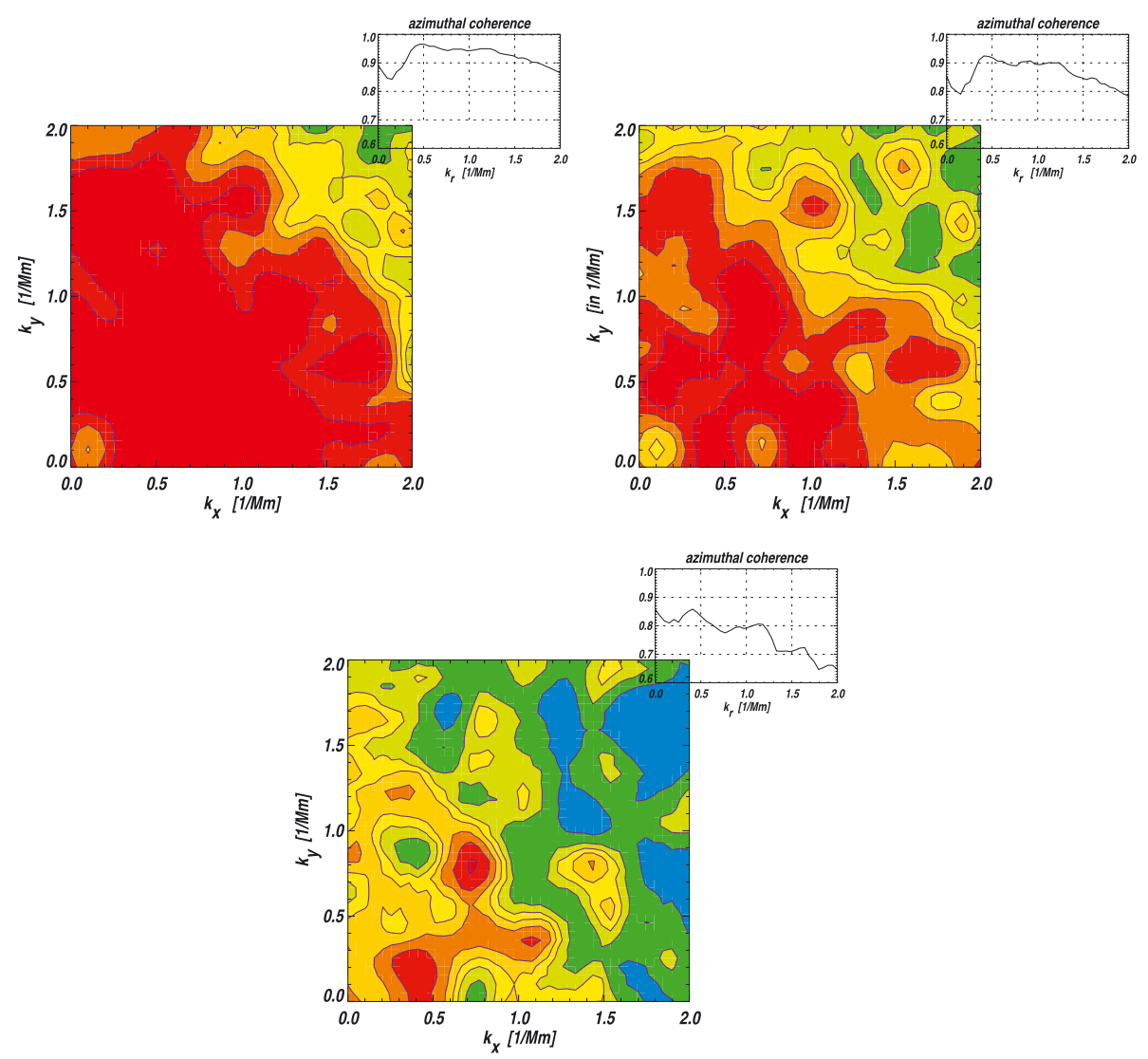

Fig. 5. Left panel: 2D coherence between velocity maps at bisector levels 0.2 and 0.5 and the associated azimuthal coherence (inset), again for the same scan 5 shown in Figs. 2-4. Right panel: same for bisector levels 0.2 and 0.75. Lower panel: same for bisector level 0.2 and line center.

In Fig. 4, lower left panel, the lines of constant cross power $Q^{(0.2,1.0)}$ do not exhibit the same gradient along the $k_{x}$ and $k_{y}$ axes, thus indicating a reflectional asymmetry with respect to the diagonal through the origin. This behavior of the cross power reflects a tendency for the dynamics to be less isotropic in the higher layers than in the lower ones.

\section{4. $2 D$ velocity squared coherence}

Figure 5 shows the results of the 2D coherence analysis applied to the four velocity maps $u^{(0.2)}, u^{(0.5)}, u^{(0.75)}$ and $u^{(1.0)}$.

The upper two panels are rather similar. They show (i) mean azimuthal coherence values of $\approx 0.8$ in the radial wavenumber interval $k_{r}[0.1-0.3] \mathrm{Mm}^{-1}$; (ii) a value $\geq 0.9$ in the $k_{r}$ interval [0.3-1.2] $\mathrm{Mm}^{-1}$; and (iii) a linear decrease down to a value $\geq 0.8$ in the [1.2-2.0] $\mathrm{Mm}^{-1}$ interval.

The lower panel shows the coherence between the $u^{(0.2)}$ and $u^{(1.0)}$ velocity maps, hence between the granular layers $(0.2)$ and the highest photospheric layers probed by the spectral line (1.0). The mean azimuthal coherence function shows a nearly linear decrease from a maximum value of $\approx 0.85$ down to $\approx 0.65$.

Generally the coherence function gives a measure of the similarity between signals and is related to the cross correlation function. More specifically, coherence is a measure of the linear dependence of one signal on another.

Therefore the mean azimuthal coherence functions (insets in Fig. 5) demonstrate the high similarity between the velocity fields measured at bisector levels 0.2 and 0.5 , and a moderate similarity between the fields at bisector levels 0.2 and 0.75 . The coherence between the velocity fields is high for radial wavenumbers $0.3 \leq k_{r} \leq 1.2 \mathrm{Mm}^{-1}$ only. This implies a high dynamical persistence of the velocity fluctuations with $k_{r} \leq 1.2 \mathrm{Mm}^{-1}$. The linear decrease of the mean azimuthal coherence between 1.0 and $2.0 \mathrm{Mm}^{-1}$ indicates the dynamical instability of the velocity fluctuations on scales smaller than $\approx 1$ arcsec. A similar finding is discussed by Nesis et al. (1997, Fig. 6).

The mean azimuthal coherence function $\left(u^{(0.2)}, u^{(1.0)}\right)$, and thus the similarity between the velocity fields of the granular layer(0.2) and the line core layer(1.0), differs in form and value from the other two azimuthal coherence functions shown in Fig. 5. The coherence function decreases practically linearly with $k_{r}$ and does not have a preferred radial wavenumber. This can be explained by the nonpersistence of velocity fluctuations of all spatial scales as they enter into the layers above those corresponding to the 0.75 bisector level. The important finding is the change of the coherence function above the 0.75 bisector level compared to the layers below. This behavior of the photospheric dynamics is found also in the power and cross-power analysis (Figs. 3 and 4). It seems that this behavior is an intrinsic property of the dynamics.

An important aspect of the coherence maps in Fig. 5 is their isotropy. In the $\left(u^{(0.2)}, u^{(0.5)}\right)$ coherence map we see a high coherence and a symmetry with respect to both axes up to wavenumbers of $\approx 1.5 \mathrm{Mm}^{-1}$. The symmetry of the $\left(u^{(0.2)}\right.$, $\left.u^{(0.75)}\right)$ coherence map is less evident and more concentrated up to $\approx 1.0 \mathrm{Mm}^{-1}$. We realize a decrease of symmetry in the $\left(u^{(0.2)}\right.$, $\left.u^{(1.0)}\right)$ coherence map, which is associated with the cross correlation between the 0.2 and line core maps. Here, the coherence contours are different above and below the diagonal through the origin of the $\left(k_{x}, k_{y}\right)$ coordinates.

To quantify the decrease of isotropy with height we correlated the coherence maps for the three upper height levels with the deepest one with their transposed maps and found correlation values of $0.6,0.5$, and 0.2 , respectively. The reduced isotropy in the higher layers is not restricted to the $\left(u^{(0.2)}, u^{(1.0)}\right)$ coherence 

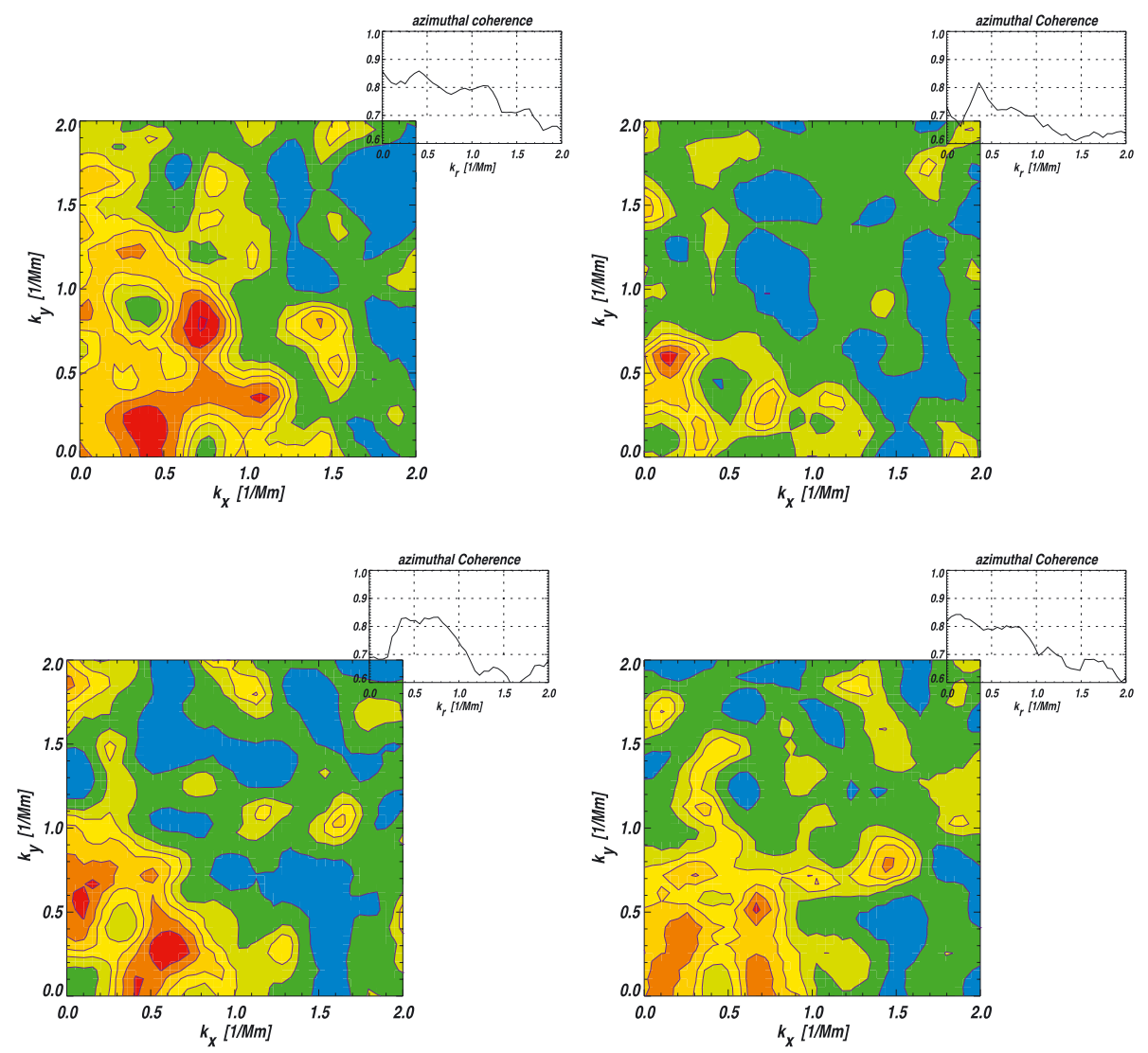

Fig. 6. Time variation of the $2 \mathrm{D}$ coherence between velocity maps at bisector levels 0.2 and line core. Four scans from the series are shown: scan 5 (upper left - same as Fig. 5, lower panel), scan 21, scan 38, and scan 72 (lower right).

Table 3. Times and correlation values.

\begin{tabular}{lcccc}
\hline \hline$\Delta$-UT [min] & 2.25 & 8.92 & 16.00 & 30.00 \\
scan & 5 & 21 & 38 & 72 \\
\hline Corr. values & 0.2 & 0.015 & 0.14 & 0.03 \\
\hline
\end{tabular}

Notes. Correlation values between the coherence maps of the highest and lowest atmospheric level and their transposed ones for the indicated time steps.

map of scan 5 (lower panel of Fig. 5), but evident at all time steps from the series, as illustrated in Fig. 6 for scans 21, 38, and 72. Table 3 lists the times at which these scans were taken as well as the correlation values between the coherence maps relating the velocity fields $\left(u^{(0.2)}, u^{(1.0)}\right)$, which are shown in Fig. 6 , and the respective transposed maps. All correlation values are seen to be very low.

This reduced isotropy of the coherence between the deepest and highest photospheric layers reflects a physical property of the photospheric flow which varies with height. According to Tennekes \& Lumley (1972) "the large-scale structure of turbulence is unlikely to be isotropic". The disappearance of small scale velocity structures with height in the photosphere (see Figs. 3 and 4) favors the coverage of the higher photospheric layers by large scale structures and causes thus the variation of the degree of isotropy between the deepest and higher layers. In this case any turbulent regions in the upper photosphere can probably not be represented by the Kolmogorov energy spectrum of homogeneous and isotropic turbulence.

The observed elongated velocity structures in the higher photospheric layers reflect the physics of an organized flow with few degrees of freedom (Nesis et al. 2001). According to Tennekes \& Lumley (1972) such a behavior might be due to the presence of magnetic fields. Even though our measurements were taken in a nonactive region, weaker intergranular magnetic fields cannot be excluded. In fact, a large body of recent numerical work on magnetoconvection (Cattaneo 1999; Vögler et al. 2007; Weiss $\&$ Proctor 2001) suggests that the solar granulation can act as dynamo source for small-scale magnetic fields. The strength of those fields is determined by the balance between the rates of vorticity generation by buoyancy and magnetic curvature forces (Galloway et al. 1978). This process might contribute to organize the flow into elongated velocity structures in the higher layers, thus reducing the number of degrees of freedom.

\section{Conclusions}

We investigated the change of the dynamical, topological and symmetry properties of the granular velocity field with height in the photosphere. The 2D power and 2D coherence analyses reveal that the velocity fields of the higher photospheric layers are different from the deeper granular layers. The loss of similarity was found to occur between the bisector levels 0.75 and 1.0, i.e. in the mid photosphere. The highest photospheric layers are characterized by (i) a diminution of the velocity power; (ii) a disappearance of small scale velocity structures; and (iii) a less isotropic behavior of the velocity fields. It should be noted that these deviations from isotropy reflect the filamentary nature of individual velocity structures and do not imply a global preferred direction. The higher photospheric layers are dominated by large-scale velocity structures $\geq 1.5 \mathrm{Mm}$ with small velocity amplitudes.

Thus, our 2D analysis demonstrates the separation of the photosphere into two parts characterized by their dynamical and morphological properties of the velocity fields. In this context the comparison with the properties of the intensity structures 
of the photospheric layers is of interest. As shown in a number of previous papers (Krieg et al. 1999; Hirzberger et al. 2001; Hirzberger 2002; Berrilli et al. 2002; Puschmann et al. 2005) granulation structures are found only up to $150 \mathrm{~km}$ above $\tau_{500}=1$.

Our results indicate that the Kolmogorov theory of isotropic turbulence cannot fully describe the dynamics of the upper photosphere, in agreement with earlier results (e.g., Petrovay 2001; Hirzberger 2002; Rieutord et al. 2010).

The physical nature of the higher photospheric layers could be dominated by gravity waves related to the decay of granular motions (Komm et al. 1991; Strous et al. 2000; Severino et al. 2003; Straus et al. 2008, 2009). Another possibility are convective upflows over the entire lifetime of granules with sometimes extreme velocities. They can be interpreted as bursts that may trigger gravity waves in the upper photosphere (Nesis et al. 2002). It is also conceivable that the dynamics of the upper photosphere traces flows that organize the spatial clustering of intensity structures in the deeper layers (Berrilli et al. 2005).

Acknowledgements. We are grateful to J. Bruls for helpful comments on the manuscript.

\section{Appendix A: 2D power and coherence analysis}

2D Fourier transform. Consider the observed velocity map as a $2 \mathrm{D}$ real sample set $\left[x_{k_{1} k_{2}}\right]$ defined on the $2 \mathrm{D}$ grid $0 \leq k_{1} \leq$ $N_{1}-1, \quad 0 \leq k_{2} \leq N_{2}-1$. The 2D discrete Fourier transform of $\left[x_{k_{1} k_{2}}\right]$ is given by $X\left(n_{1}, n_{2}\right) \equiv X_{n_{1} n_{2}}$, where $n_{1}, n_{2}$ are the spatial frequencies or wave-numbers on the same grid,

$X_{n_{1} n_{2}}=\sum_{k_{1}=0}^{N_{1}-1} \sum_{k_{2}=0}^{N_{2}-1} x_{k_{1} k_{2}} \exp \left(2 \pi \mathrm{i} k_{1} n_{1} / N_{1}\right) \exp \left(2 \pi \mathrm{i} k_{2} n_{2} / N_{2}\right)$

where $-\frac{N_{1}}{2} \leq n_{1} \leq \frac{N_{1}}{2}$ and $-\frac{N_{2}}{2} \leq n_{2} \leq \frac{N_{2}}{2}$.

2D periodogram (2D power spectrum). Given a real velocity sample set $\left[x_{k_{1} k_{2}}\right]$ as e.g. the one in the above paragraph, the periodogram is defined as the squared magnitude of the discrete Fourier transform (DFT) of $\left[x_{k_{1} k_{2}}\right]$ usually scaled by $N_{1} \times N_{2}$, the number of samples in $\left[x_{k_{1} k_{2}}\right]$. Thus from Eq. (A.1), assuming $N_{1}$ and $N_{2}$ are even, we define the periodogram $P_{n_{1} n_{2}}$ as a collection of $\left(\frac{N_{1}}{2}+1\right) \times\left(\frac{N_{2}}{2}+1\right)$ real values:

$P_{n_{1} n_{2}} \triangleq$ periodogram of $\left[x_{k_{1} k_{2}}\right] \triangleq \frac{1}{N_{1} N_{2}}\left|X_{n_{1} n_{2}}\right|^{2}$,

where we restrict ourselves to $0 \leq n_{1} \leq \frac{N_{1}}{2}, 0 \leq n_{2} \leq \frac{N_{2}}{2}$ because the measured quantity is real valued and the other quadrants yield the same information: according to Eq. (A.1) if $\left[x_{k_{1} k_{2}}\right]$ is a real 2D spatial sample set then $P_{n_{1} n_{2}}$ is a symmetric function, i.e. $P_{n_{1} n_{2}}=P_{\left(N_{1}-n_{1}\right)\left(N_{2}-n_{2}\right)}$ for $n_{1}$ and $n_{2}$ ranging from $\frac{N_{1}}{2}+1, \frac{N_{2}}{2}+1$ to $N_{1}-1$ and $=N_{2}-1$, respectively.

According to Parseval's relation one obtains for the total power of the data field $\left[x_{k_{1} k_{2}}\right]$

$$
\begin{aligned}
E\left[x_{k_{1} k_{2}}^{2}\right] & =\frac{1}{\left(N_{1} N_{2}\right)^{2}} \sum_{n_{1}=0}^{N_{1}-1} \sum_{n_{2}=0}^{N_{2}-1}\left|X_{n_{1} n_{2}}\right|^{2} \\
& =\frac{1}{N_{1} N_{2}} \sum_{n_{1}=0}^{N_{1}-1} \sum_{n_{2}=0}^{N_{2}-1} P_{n_{1} n_{2}},
\end{aligned}
$$

where $k_{1}=0, \ldots, N_{1}-1, \quad k_{2}=0, \ldots, N_{2}-1$.
2D cross periodogram (2D cross power spectrum). Equation (A.2) shows that the power density spectrum of velocity map $\left[x_{k_{1} k_{2}}\right]$ is the average squared magnitude of the DFT. The cross power density spectrum, or cross spectrum in short, is similar but involves two velocity maps $\left[x_{k_{1} k_{2}}\right]$ and $\left[y_{k_{1} k_{2}}\right]$ instead of just $\left[x_{k_{1} k_{2}}\right]$. The estimated cross power spectrum is the average of DFT products of the form

$$
\begin{aligned}
I_{x y} \equiv I_{n_{1} n_{2}} & \triangleq \text { cross periodogram of }\left[x_{k_{1} k_{2}}, y_{k_{1} k_{2}}\right] \\
I_{n_{1} n_{2}} & =\frac{1}{\left(N_{1} N_{2}\right)} X_{n_{1} n_{2}}^{*} Y_{n_{1} n_{2}},
\end{aligned}
$$

where $0 \leq n_{1} \leq \frac{N_{1}}{2}, \quad 0 \leq n_{2} \leq \frac{N_{2}}{2}$. The asterisk $\left(^{*}\right)$ denotes conjugate. Unlike $P_{n_{1} n_{2}}$ in Eq. (A.2), $I_{n_{1} n_{2}}$ in Eq. (A.4) is a complex quantity with $I_{n_{1} n_{2}}=I_{\left(N_{1}-n_{1}\right)\left(N_{2}-n_{2}\right)}^{*}$ for $n_{1}$ from $\frac{N_{1}}{2}+1$ to $N_{1}-1$, and $n_{2}$ from $\frac{N_{2}}{2}+1$ to $N_{2}-1$.

The expected total cross power of $\left[x_{k_{1} k_{2}}, y_{k_{1} k_{2}}\right]$ is given by

$$
\begin{aligned}
E\left[x_{k_{1} k_{2}}, y_{k_{1} k_{2}}\right] & =\frac{1}{\left(N_{1} N_{2}\right)^{2}} \sum_{n_{1}=0}^{N_{1}-1} \sum_{n_{2}=0}^{N_{2}-1} \operatorname{Re}\left[X_{n_{1} n_{2}}^{*} Y_{n_{1} n_{2}}\right] \\
& =\frac{1}{\left(N_{1} N_{2}\right)} \sum_{n_{1}=0}^{N_{1}-1} \sum_{n_{2}=0}^{N_{2}-1} \operatorname{Re}\left[I_{n_{1} n_{2}}\right] .
\end{aligned}
$$

The 2D cross periodogram $I_{n_{1} n_{2}}$ is a measure of the cross power between two velocity maps $\left[x_{k_{1} k_{2}}\right]$ and $\left[y_{k_{1} k_{2}}\right]$, or in other words the power that $\left[x_{k_{1} k_{2}}\right]$ and $\left[y_{k_{1} k_{2}}\right]$ have in common at specific frequencies.

2D cross spectrum. The cross spectrum $Q_{n_{1} n_{2}}$ is defined as follows:

$Q_{n_{1} n_{2}}=\operatorname{Re}\left[I_{n_{1} n_{2}}\right]=\operatorname{Re}\left[X_{n_{1} n_{2}}^{*} Y_{n_{1} n_{2}}\right]$

where $0 \leq n_{1} \leq \frac{N_{1}}{2}, 0 \leq n_{2} \leq \frac{N_{2}}{2}$.

$2 \mathrm{D}$ cross phase spectrum. The quadrature spectrum $q_{n_{1}, n_{2}}$ is defined as follows:

$q_{n_{1} n_{2}}=\operatorname{Im}\left[I_{n_{1} n_{2}}\right]=\operatorname{Im}\left[X_{n_{1} n_{2}}^{*} Y_{n_{1} n_{2}}\right]$

where $0 \leq n_{1} \leq \frac{N_{1}}{2}, 0 \leq n_{2} \leq \frac{N_{2}}{2}$.

Considering the two observed velocity maps $x_{k_{1} k_{2}}$ and $y_{k_{1} k_{2}}$ as real functions defined over the same $2 \mathrm{D}$ grid $0 \leq k_{1} \leq N_{1}-1$, $0 \leq k_{2} \leq N_{2}-1$, we can define their 2D coherence as a function $\Gamma_{x y}\left(n_{1}, n_{2}\right)$. The $2 \mathrm{D}$ magnitude squared coherence of the $x_{k_{1} k_{2}}$ and $y_{k_{1} k_{2}}$ maps is the normalized version of their $2 \mathrm{D}$ cross power and is defined as follows:

$\Gamma_{x y}^{2}\left(n_{1}, n_{2}\right)=\frac{\left|X_{n_{1} n_{2}} Y_{n_{1} n_{2}}^{*}\right|^{2}}{\left|X_{n_{1} n_{2}}\right|^{2}\left|Y_{n_{1} n_{2}}\right|^{2}}$,

where cross power $\left|X_{n_{1} n_{2}} Y_{n_{1} n_{2}}^{*}\right|$ and auto power $\left|X_{n_{1} n_{2}}\right|^{2},\left|Y_{n_{1} n_{2}}\right|^{2}$ are related to cross correlation and autocorrelation as follows: cross correlation $(x, y) \leftrightarrow\left(X_{n_{1} n_{2}}\right)\left(Y_{n_{1} n_{2}}^{*}\right) \quad$ correlation theorem auto correlation $(x, x) \leftrightarrow\left|X_{n_{1}, n_{2}}\right|^{2} \quad$ Wiener-Khintchine theorem.

\section{References}

Berrilli, F., Consolini, G., Pietropaolo, E., et al. 2002, A\&A, 381, 253 Berrilli, F., Del Moro, D., Russo, S., Consolini, G., \& Straus, Th. 2005, ApJ, 632, 677 
A. Nesis et al.: Solar photospheric velocity patterns

Canfield, R. C., \& Mehltretter, J. P. 1973, Sol. Phys., 33, 33

Cattaneo, F. 1999, ApJ, 515, L39

Espagnet, O., Muller, R., Roudier, Th., \& Mein, N. 1993, A\&A, 271, 589

Espagnet, O., Muller, R., Roudier, Th., Mein, N., \& Mein, P. 1995, A\&AS, 109, 79

Galloway, D. J., Proctor, M. R. E., \& Weiss, N. O. 1978, J. Fluid Mech., 87, 243

Hanslmeier, A., Kučera, A., Rybák, J., Neunteufel, B., \& Wöhl, H. 2000, A\&A, 356,308

Hirzberger, J. 2002, A\&A, 392, 1105

Hirzberger, J., Koschinsky, M., Kneer, F., \& Ritter, C. 2001, A\&A, 367, 1011

Komm, R., Mattig, W., \& Nesis, A. 1990, A\&A, 239, 340

Komm, R., Mattig, W., \& Nesis, A. 1991, A\&A, 252, 827

Krieg, I., Wunnenberg, M., Kneer, F., Koschinsky, M., \& Ritter, C. 1999, A\&A, 343, 983

Nesis, A. 1985, Ph.D. Thesis, TU Berlin

Nesis, A., \& Mattig, W. 1989, A\&A, 221, 130

Nesis, A., Hammer, R., Hanslmeier, A., et al. 1997, A\&A, 326, 851

Nesis, A., Hammer, R., Roth, M., \& Schleicher, H. 2001, A\&A, 373, 307

Nesis, A., Hammer, R., Roth, M., \& Schleicher, H. 2002, A\&A, 396, 1003

Petrovay, K. 2001, Space Sci. Rev., 95, 9

Priestley, M. B. 1999, Spectral Analysis and Time Series (San Diego: Academic Press
Puschmann, K. G., Ruiz Cobo, B., Vazquez, M., Bonet, J. A., \& Hanslmeier, A. 2005, A\&A, 441, 1157

Rieutord., M., Roudier, T., Rincon, F., et al. 2010, A\&A, 512, A4

Roudier, Th., \& Muller, R. 1986, Sol. Phys., 107, 11

Roudier, Th., Vigneau, J., Espagnet, O., et al. 1991, A\&A, 248, 245

Stearns, S. D., \& David, R. A. 1993, Signal Processing Algorithms in Fortran and C (Englewood Cliffs: P T R Prentice-Hall)

Severino, G., Oliviero, M., Straus, Th., \& Ulrich, R. K. 2003, Mem. S. A. It., 74, 595

Straus, Th., Fleck, B., Jefferies, S. M., et al. 2008, ApJ, 681, L125

Straus, Th., Fleck, B., Jefferies, S. M., et al. 2009, 2nd Hinode Science Meeting, ed. B. Lites, M. Cheung, T. Magara, et al., ASP Conf. Ser., 415, 95

Strous, L. H., Goode, P. R., \& Rimmele, T. R. 2000, ApJ, 535, 1000

Tennekes, H., \& Lumley, J. L. 1972, A First Course in Turbulence (Cambridge, Mass.: MIT Press)

Tritschler, A., Schmidt, W., Langhans, K., \& Kentischer, T. 2002, Sol. Phys. 211, 17

Vögler, A., \& Schüssler, M. 2007, A\&A, 465, L43

Weiss, N. O., \& Proctor, M. R. E. 2001, in Recent Insights into the Physics of the Sun and Heliosphere: Highlights from SOHO and other Space Missions, ed. P. Brekke, B. Fleck, \& J. Gurman (San Francisco: ASP), IAU Symp., 203, 219 